\title{
The Benefits and Applications of a CMOS-based EBSD Detector
}

\author{
J. Goulden ${ }^{1}$, P. Trimby ${ }^{1}$, A. Bewick ${ }^{1}$ \\ ${ }^{1}$ Oxford Instruments NanoAnalysis, Halifax Road, High Wycombe, UK
}

CCD-based EBSD detectors have been commercially available for more than 15 years. These detectors are typically either 'high resolution' or 'high speed'. The 'high resolution' detectors enable the acquisition of high-quality, megapixel-resolution $(>1024 \times 1024$ pixels $)$ diffraction patterns with good sensitivity, but limited acquisition speed. Alternatively, 'high speed' detectors, typically offer a VGA resolution (maximum 640x480 pixels), with much higher maximum speeds. The latest high-speed CCDbased detectors achieve speeds of 1500 patterns per second (pps), but only by using extreme pixel binning. This binning has two undesirable effects with regard to pattern quality: a reduction in resolution (only $\sim 40 \times 30$ pixels at the highest speed); and a reduction in the maximum signal-to-noise ratio (SNR) before pixel saturation. As a result this high pattern binning compromises analysis, reducing both the hit rate and the orientation precision of fast data acquisition. Not all EBSD applications require a detector's maximum speed. Some applications require high sensitivity, high resolution and high pattern quality.

The development of a CMOS sensor for EBSD detectors has overcome these issues, delivering a step change in performance. The CMOS sensor architecture enables high speed without the need for extreme, on-sensor binning.

Therefore good resolution and good maximum SNR can be achieved during fast data acquisition. Results from a CMOS-based detector can be collected and analysed in real time at speeds in excess of 3000pps, more than double the speed of any existing CCD-based detector. Importantly, this CMOS detector has a superior maximum SNR and 4x the resolution (16x more pixels) at $>3000$ pps than a CCD detector at $\sim 1500$ pps (Figure 1). As a result this technology delivers significant improvements to both indexing hit rates and to the angular resolution of orientation measurements.

Optimising angular resolution is important in many EBSD applications, resulting in a significant interest in high resolution (HR) EBSD. This technique uses pattern correlation techniques to compare acquired patterns to reference patterns and to calculate small diffraction pattern shifts [1]. This has enabled analyses with significantly improved angular resolution, when compared to standard Hough-based indexing, with angular accuracies routinely reported below $0.01^{\circ}$. Further recent advances have explored the potential of "forward modelling" in EBSD, involving generating simulated patterns close to each measured orientation and utilising image correlation techniques to refine the orientation measurement [2, 3]. This has proved particularly valuable for dictionary-based indexing, and offers significant potential for further improvements to Hough-based indexing.

However, these approaches to improving angular resolution generally require significant time consuming, offline data processing. This involves saving multiple diffraction patterns, generating simulated patterns followed by subsequent image correlation and analysis.

In this paper we investigate real-time data acquisition approaches to determine the limits of both speed and angular accuracy using CMOS EBSD technology, enabling a combination of both high pattern 
resolution AND high speed. This offers potential improvements in angular orientation with real time indexing. Using this detector, the angular accuracy is demonstrated by both conventional Hough based indexing and a real-time solution refinement approach applied within the acquisition software.

The results indicate that there is an underestimation in the literature of the angular accuracy of real-time EBSD indexing. Tests on a silicon single crystal show that an angular resolution $<0.05^{\circ}$ is easily achievable at speeds in excess of 80 patterns per second (pps) using EBSP resolutions of $622 \times 512$ pixels, as shown in figure 2. Even further improvements in accuracy can be achieved using megapixel resolution patterns with slower frame rates. It is also notable that at high speeds ( $>2000$ pps) angular resolutions better than $0.2^{\circ}$ are still achievable.

The performance advantages offered by CMOS technology will be reviewed with examples particularly with respect to high angular resolution EBSD.

\section{References:}

[1] Wilkinson, A. J., Meaden, G. \& Dingley, D. J. Mater. Sci.Technol. 22 (2006), p. 1271.

[2] Singh, S., Ram, F. \& de Graef, M. J. Appl. Cryst. 50 (2017).

[3] Alkorta, J., Marteleur, M. \& Jacques, P.J. Ultramicr. 182 (2017), p. 17.



40 x 30 pixel EBSP collected at 1580 pps using a conventional CCD-based detector
Figure 1. Example EBSPs

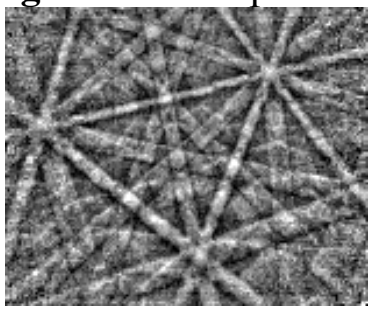

$156 \times 128$ pixel EBSP collected at 3000 pps using

CMOS-based detector

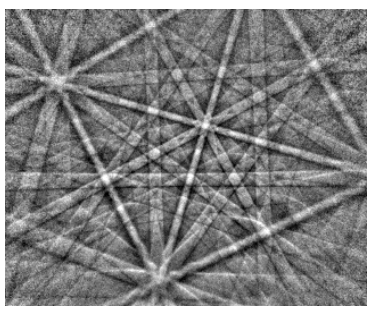

1244 x 1024 pixel EBSP collected at $100 \mathrm{pps}$ from a duplex steel sample using a CMOS-based detector

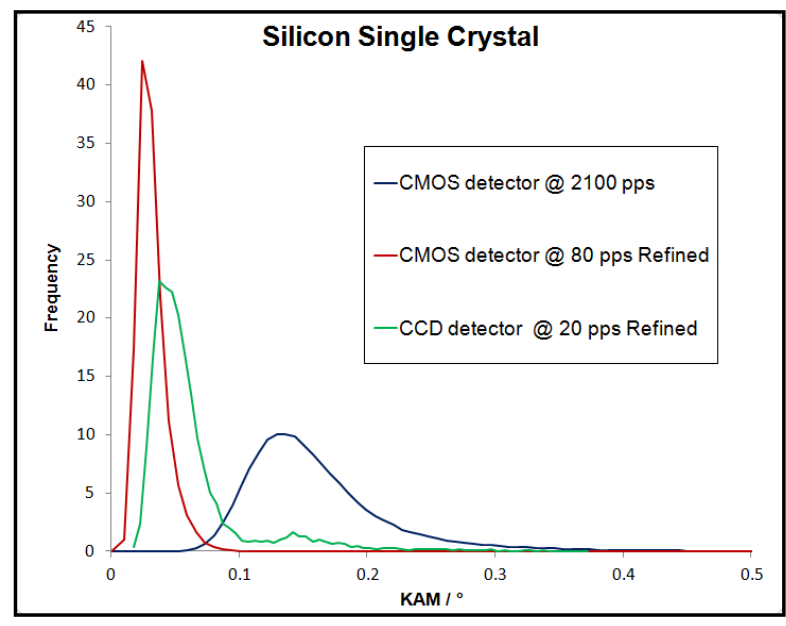

Figure 2. Kernel Average Misorientation distribution plots showing angular accuracy of different analyses / detectors on a Si single crystal. Note the significant improvement in speed and accuracy of a CMOS detector when compared to a $\mathrm{CCD}$ detector. 